\title{
Investigation of damage detectability in composites using frequency-based classification of acoustic emission measurements
}

\author{
Sebastian Felix Wirtz (D, Nejra Beganovic and Dirk Söffker
}

\begin{abstract}
Advances in composite technology led to the substitution of conventional, metallic construction material by composites. However, the more widespread application of composites is currently restricted by complex fracture mechanisms, which are not well understood. One approach to overcome this challenge is structural health monitoring systems which provide a lot of information on the current system state as well as state of health in real time. In this context, reliability assessment of structural health monitoring systems is currently an open issue. The reliability of conventional nondestructive testing systems is evaluated, measured, and partly standardized using widely accepted methods such as the probability of detection rate. Frequently, the $a_{90 \mid 95}$ value, which is determined from the probability of detection curves, is used as a performance measure indicating the minimum damage size that is detected with a probability of $90 \%$ and $95 \%$ confidence. In contrast to non-destructive testing, structural health monitoring involves additional data analysis steps, that is, statistical pattern recognition, where the classification results are also subject to uncertainty. Because similar methods are not available, the reliability of structural health monitoring systems is usually not quantified. To investigate the influences on the classification performance, experiments were conducted. In particular, the effect of variable loading conditions and the evolution of damage over time are considered. To this end, acoustic emission measurements were performed, while the specimens of the composite material were subjected to different cyclic loading patterns. Here, acoustic emission refers to elastic stress waves in the ultrasound regime, which emerge from the structure on damage initiation and propagation. Furthermore, a frequency-based damage classification scheme for acoustic emission measurements is proposed. Time-frequency domain features are extracted from the measurement signals using shorttime Fourier transform. Classification is performed using support vector machine. Both choices serve as typical examples to discuss the effects which apply equally to other approaches. Experimental results presented in this article regarding fault diagnosis and discrimination of delamination, matrix crack, debonding, and fiber breakage in carbon-fiberreinforced polymer material show that good performance applying support vector machine could be achieved using 10 fold cross validation. However, during model deployment, strong dependency of the classification reliability on loading conditions can be clearly stated, which could not be seen from the previous evaluation. Concluding from these results, it can be stated that the application of classifier-based structural health monitoring is more complex than generally assumed. The relations between the classification approaches, testing conditions, measurement devices, and filters have to be discussed with respect to the ability to provide reliable statements about the actual damage state.
\end{abstract}

\section{Keywords}

Acoustic emission (AE), support vector machine (SVM), composite material, probability of detection (POD), reliability, classification, non-destructive testing (NDT)

\section{Introduction}

The recent rise of composites is owed to their beneficial properties, such as fatigue strength, impact resistance, and lightweight, resulting from their sophisticated structure. Today, the more widespread use of
Chair of Dynamics and Control, University of Duisburg-Essen, Duisburg, Germany

Corresponding author:

Dirk Söffker, Chair of Dynamics and Control, University of DuisburgEssen, 47057 Duisburg, Germany.

Email: soeffker@uni-due.de 
composites is restricted for several reasons. Compared to metallic materials, composites lack the pronounced ductile behavior. ${ }^{1}$ Furthermore, composites form a complex system defined by the constituent materials' properties, geometry, and distribution, whereas metals are generally assumed to exhibit homogeneous mechanical properties. ${ }^{2}$ Therefore, complex micro-mechanical fracture mechanisms are observed. ${ }^{1}$ Structural health monitoring (SHM) systems are proposed to overcome these challenges and to ensure equal safety and reliability of composite structures. ${ }^{3}$ This includes the fact that the SHM system applied has to ensure reliable measurements and conclusions regarding the actual system state.

A definition of SHM is given by Farrar and Worden $^{4}$ as "the process of implementing a damage identification strategy for aerospace, civil, and mechanical engineering." In general, the goal of such a strategy is to establish a surveillance system that is capable of continuously monitoring a technical system or structure. This enables advanced maintenance strategies, that is, condition-based maintenance, which leads to an increase in reliability of technical systems. ${ }^{5}$ In this context, methods for non-destructive evaluation (NDE) are employed. In particular, acoustic emission (AE) technique, which is a passive, wave-propagation-based NDE method, gained attention for in situ monitoring recently. In general, AE refers to the phenomenon of elastic waves generated in the ultrasound regime due to the sudden release of energy. These elastic waves emerge from distinct sources within a structure at frequencies between $10 \mathrm{kHz}$ and $1 \mathrm{MHz} .^{6}$ In particular, this occurs on the initiation and propagation of damage or due to external impact loads. Consequently, AE monitoring should be applied while the structure is loaded.

Sources of AE are manifold. In composites, only distinct types of damage are observed as a result of the underlying micro-mechanical fracture mechanisms. In particular, these are delamination, matrix crack, fiber breakage, and debonding. ${ }^{7}$ Whereas debonding merely describes the loss of adhesion between fiber and matrix material, delamination denotes the separation of layers in laminated materials. ${ }^{3}$ The resulting AE waveforms are characteristic to the particular source mechanism and, hence, AE measurements can be utilized to identify the corresponding fracture mechanism, which has already been shown in several case studies. ${ }^{3,8-11}$ For this purpose, modal analysis and time- and frequencydomain-based approaches are distinguished.

Regarding the modal properties of the AE waveforms, the corresponding fracture mechanisms can be identified based on physical interpretation of the source motion. In thin plates, these waveforms propagate as guided waves, which can be described by means of
Lamb wave theory. ${ }^{11}$ Accordingly, two distinct wave modes - flexural and extensional waves - exist. These are promoted by either in-plane or out-of-plane source motion, respectively. ${ }^{12}$ According to Prosser $^{12}$ and Gutkin et al., ${ }^{13}$ the extensional wave mode is usually observed at higher frequencies and exhibits faster propagation velocities than flexural waves. Furthermore, this wave mode is symmetric and non-dispersive. In contrast, flexural waves are antisymmetric, propagate at lower velocities, and are highly dispersive. In general agreement, several authors reported in-plane motion to be associated with fiber breakage ${ }^{3,9}$ and matrix crack. ${ }^{9,11}$ These damage mechanisms promote highfrequency extensional waves. In contrast, delamination is governed by out-of-plane motion and thus promotes the flexural waves in the material generating lowfrequency signals. ${ }^{3,9,11}$

For the purpose of automated damage classification, several statistical properties of the AE signals - referred to as features or descriptors - that can be calculated from both time and frequency domains, are frequently used. Typically, time domain features are used for the analysis of AE measurements. ${ }^{13-15}$ However, time domain features are strongly dependent on the experimental conditions, whereas the frequency content is not affected. Particularly, the AE amplitude is subject to variable attenuation depending on the propagation path. ${ }^{11}$ Thus, the frequency spectrum of AE signals is considered a more reliable descriptor of AE sources. ${ }^{9}$

To identify characteristic frequencies of distinct $\mathrm{AE}$ source mechanisms, peak frequency analysis was applied by several researchers. ${ }^{10,16,17}$ De Groot et al. ${ }^{16}$ identified damage-specific signatures of four different micro-mechanical damage modes, namely, matrix crack [90 kHz, $100 \mathrm{kHz}$ ], debonding [240 kHz, $310 \mathrm{kHz}$, fiber breakage $[>300 \mathrm{kHz}]$, and fiber pull-out [180 kHz, $240 \mathrm{kHz}]$ in carbon-fiber-reinforced polymer (CFRP) material in terms of peak frequencies. Similarly, Hamdi et al. $^{10}$ identified delamination [30 kHz, $90 \mathrm{kHz}$, matrix crack [30 kHz, $170 \mathrm{kHz}$, debonding $[180 \mathrm{kHz}, 290 \mathrm{kHz}]$, and fiber breakage [300 kHz, $420 \mathrm{kHz}]$ as distinct classes of micromechanical damage in composites using HilbertHuang transform (HHT).

To identify characteristic peak frequencies and to track damage accumulation under different experimental conditions, Bussiba et al. ${ }^{17}$ used short-time Fourier transform (STFT). Based on their experimental results, three characteristic frequencies were identified, which correspond to the damage mechanisms matrix such as crack $(140 \mathrm{kHz})$, debonding $(300 \mathrm{kHz})$, and fiber breakage $(405 \mathrm{kHz})$. Moreover, mechanical thresholds for the onset of $\mathrm{AE}$ activity were determined, indicating that no damage occurs below these threshold values. ${ }^{17}$ 
Damage characterization task is most frequently considered as the classification problem. Pattern recognition algorithms are a suitable method to address this type of problem. ${ }^{18}$ Here, statistical learning theory is employed to determine the mapping between class labels and input values. In the supervised case, a representative set of training data are used to generate the statistical model. The most frequently used classifier algorithms are $K$-nearest neighbor $(\mathrm{KNN})$, artificial neural network (ANN), and support vector machine (SVM). Das et al. ${ }^{19}$ stated that SVM is a suitable method to identify damage modes in composites. Here, joint time-frequency transformation was performed prior to the classification to extract damage-specific features.

A generic SHM system is composed of a measurement chain, where principles of non-destructive testing (NDT) are employed, and a signal processing chain. According to the statistical pattern recognition paradigm, damages are detected by means of classification. ${ }^{4}$ To realize SHM systems in practice, a suitable and therefore well-defined reliability of the classification must be achieved. This includes high detection rates as well as low false alarm rates, so that the system can be accepted. Furthermore, the surveillance system should be robust against external disturbances.

The reliability of conventional NDT methods is frequently assessed using probability of detection (POD) as a probabilistic approach, which provides a measure of the reliability of an NDT method. ${ }^{20}$ The POD curve describes the likelihood that a certain flaw is detected as a function of flaw characteristic $a$ such as size or depth. These POD curves can be computed directly from the experimental data, where two approaches are distinguished. In the case of binary response of the inspection system, hit/miss analysis is employed, whereas $\hat{a}$ vs $a$ approach can be used if the continuous output $\hat{a}$ of the inspection system is available. ${ }^{20}$ Commonly, the $a_{90 \mid 95}$ value is determined from the POD curve as a performance measure of the inspection system. ${ }^{21}$

The performance evaluation of a classifier is usually based on a set of test data with known class labels. Here, the classifier output and true class labels are compared by means of a confusion matrix. From the confusion matrix, different scores, such as accuracy, sensitivity, and specificity, are extracted to assess the performance of classification algorithms. ${ }^{22}$ Here, sensitivity denotes the detection rate, whereas false alarm rate is the complement of the specificity of a classifier. In general, improved detection rates can only be achieved at the cost of increasing false alarm rates. The principle relationship between the detection and false alarm rates is described by receiver operating characteristic (ROC) curve, ${ }^{23}$ which compares the detection and false alarm rates of a classifier. In the context of classification algorithms, the POD is understood as the true-positive rate, which is also known as the sensitivity of a classifier. ${ }^{22,24}$ However, these measures only provide quantification of model performance with respect to a specific set of testing data.

Due to conceptual differences between SHM and NDE, reliability of SHM systems is usually not quantified. In order to determine the POD curve of an NDE inspection technique, the fixed decision threshold of the sensor response $\hat{a}$ is determined using model (calibration) specimens under controlled laboratory conditions. ${ }^{25}$ Due to the in-service application of SHM, damages evolve over time and exclusion of disturbances is generally not possible. ${ }^{26}$ Consequently, the sensor output is compared to a baseline signal for damage detection, where deviations cannot be readily attributed to damage due to in situ effects and hence require appropriate interpretation. ${ }^{25}$ Influencing factors of NDE systems are, for instance, reported as testing equipment and procedures, material and geometry of test specimens, and properties of the particular defect. ${ }^{20}$ In contrast to this, SHM systems are reportedly affected by loading conditions, ${ }^{26}$ temperature, ${ }^{25}$ and sensor degradation. ${ }^{25}$ For instance, Gagar et al. ${ }^{27}$ reported a strong dependence of $\mathrm{AE}$ activity on the particular loading conditions using aluminum specimens under cyclic loading patterns. Furthermore, Schubert Kabban et al. ${ }^{21}$ mentioned that the assumption of independent observations is not feasible in case of SHM systems, because measurements are performed at high acquisition rates to determine the current state of health in real time leading to several dependent observations.

In this work, an experimental study regarding the impact of different loading conditions on the reliability of supervised classifiers is presented. Due to practical relevance, diagnosis of a composite structure was chosen to showcase SHM implementation. A mechanical test rig was used to simulate a load-bearing structure of composite material, while AE measurements are performed. Furthermore, a statistical pattern recognition approach using STFT-based feature extraction and SVM-based classification of the measurement results is proposed as an example. The performance of the classifier is evaluated using 10 -fold cross validation, which is a widely accepted approach in the field of machine learning for evaluating classifiers. Finally, the reliability of the classification results obtained from the deployed model is evaluated with respect to damage evolution and variable loading conditions using probability estimation. This result is new and could not be detected from previous publications. In the following sections, the experimental procedure is introduced, the measuring chain and the employed signal processing techniques are described. In the following, the experimental results of the proposed procedure are presented and 
discussed with respect to reliability considerations of SHM applications. Finally, the main conclusions from the experimental results are summarized.

\section{Experiments}

In the context of this work, experiments were performed to investigate the effect of loading conditions and damage evolution on the reliability of automatic damage classification. To this end, a test rig is used to subject specimens of composite material to cyclic loading patterns, while AE measurements are performed for diagnostic purposes. In this section, the mechanical test rig, data acquisition hardware, and methods used for signal processing are briefly described.

\section{Mechanical test rig}

In order to simulate an in-service, load-bearing structure, a mechanical test rig was developed, which is used to subject specimens of composite material to cyclic loading patterns. The major components of the test rig are presented in Figure 1.

The frame construction of the test rig consists of aluminum profiles, having a vise attached to fixate the specimen during testing. Furthermore, a slider-crank mechanism, which converts the rotational motion of the motor into linear displacement, is used to apply bending load by deflecting the specimen tip. For actuation of the test rig, a servo-controlled brushless direct current (BLDC) motor manufactured by Maxon motors is mounted on the aluminum frame. The BLDC motor is driven by a servo-amplifier of the type Maxon 4-Q-EC, providing control of the motor current. Setpoint values for the motor current are read from analog input in the range of $0-5 \mathrm{~V}$. Moreover, a laser proximity sensor of the type ODSL9 by Leuze is used

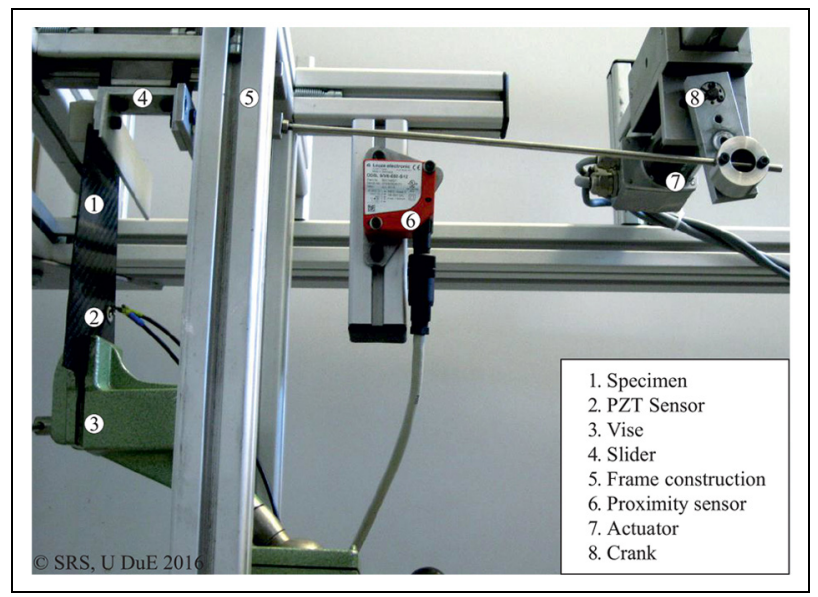

Figure I. Components of the mechanical test rig, SRS U DuE. for contactless displacement measurement. The sensor provides a maximum resolution of $0.1 \mathrm{~mm}$ in the maximum measuring range of $50-650 \mathrm{~mm}$ of distance. Here, the measuring range was configured to an interval of $65 \mathrm{~mm}$. The motion control algorithm is implemented using LabView and a National Instruments USB I/O board of the type NI USB 6229, featuring 32 analog input and 4 analog output ports of 16 bit resolution. To drive the actuator, output values are computed by the control algorithm according to the actual displacement of the tip, which is captured by the laser proximity sensor.

\section{Data acquisition}

The AE technique relies on the measurement of surface waves, such as Rayleigh and Lamb waves. ${ }^{28}$ Therefore, small surface displacements need to be detected to record AE. Consequently, signals obtained from AE measurements are characterized by high frequency content and low amplitudes. Hence, high sensitivity of the measuring system and high acquisition rates are crucial. ${ }^{12}$ For instance, Al-Jumaili et al. ${ }^{29}$ used a sampling rate of $5 \mathrm{MHz}$ for AE monitoring.

A generic measuring chain for $\mathrm{AE}$ applications consists of sensors, amplifiers, and data acquisition hardware. ${ }^{6}$ To record surface waves generated by $\mathrm{AE}$, a surface-mounted, piezoelectric acceleration sensor is employed, because it is a robust and well-established technology in the field of AE. It consists of a diskshaped piezoceramic element of $0.55 \mathrm{~mm}$ thickness and $\varnothing 10 \mathrm{~mm}$ in diameter featuring a resonant frequency of 3.6 MHz. As the bonding agent, cyanoacrylic glue was used to attach the sensor to the specimen. This couplant was reported to provide good reproducibility compared with other couplants. ${ }^{30}$ Furthermore, the stiff bonding improves transmission properties of in-plane wave modes and provides permanent bonding of the sensor to the structure. ${ }^{31}$ To capture the low-power sensor response produced by the piezoelectric element, the sensor is connected to a pre-amplification device which drives the A/D conversion hardware. For data acquisition, a field-programmable gate array (FPGA) board offering 16 bit resolution at a maximum sampling rate of $25 \mathrm{MHz}$ is used. A sampling rate of $4 \mathrm{MHz}$ was chosen as the suitable trade-off between resolution and technical requirements. The waveforms were acquired continuously.

Examples of the acquired waveforms are presented in Figures 2 and 3, respectively. Here, the time series data as well as joint time-frequency domain representation using continuous wavelet decomposition are presented. The event shown in Figure 2 is considered as representative of delamination. The source motion of this fracture mechanism is mainly characterized by 


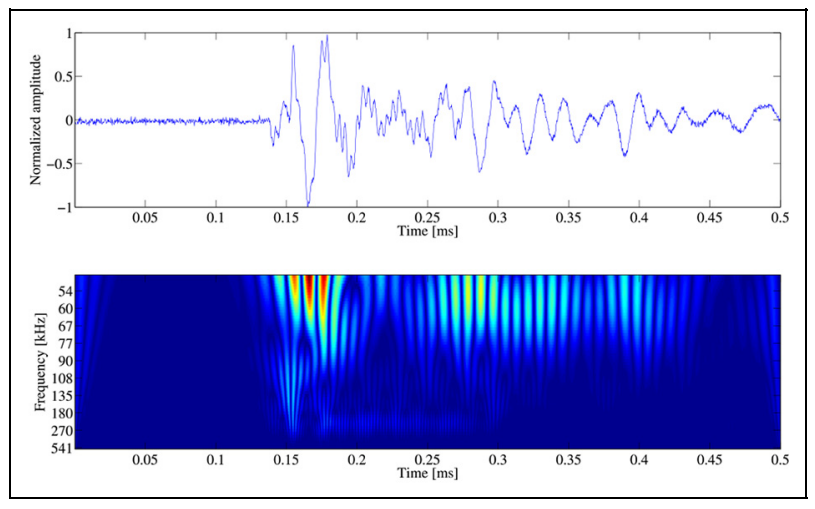

Figure 2. Time and time-frequency representation of delamination events.

out-of-plane displacement. According to the literature, AE events of high amplitude exhibiting a dominant flexural wave mode are presumably associated with delamination. ${ }^{3,9,11}$ Furthermore, these waveforms are highly dispersive and show long durations. ${ }^{12}$ In general agreement, the frequency content of delamination is reported in the lower frequency band of the ultrasonic regime at the frequencies of $[50 \mathrm{kHz}, 150 \mathrm{kHz}]$ according to Gutkin et al., ${ }^{13}$ whereas Hamdi et al. ${ }^{10}$ reported lower frequencies of delamination events in the range of $[30 \mathrm{kHz}, 90 \mathrm{kHz}]$. In contrast to this, the $\mathrm{AE}$ waveform presented in Figure 3 is attributed to the class of fiber breakage. This type of damage occurs, if the maximum strain of the fiber is exceeded due to excessive deformation of the matrix material. Here, the rapid redistribution of stress due to the reinforcement failure primarily activates in-plane source motion. Therefore, high-frequency extensional modes featuring short rise time and duration are associated with fiber breakage., ${ }^{3,9}$ According to the literature, the peak frequency is localized at frequencies above $300 \mathrm{kHz}$. The maximum

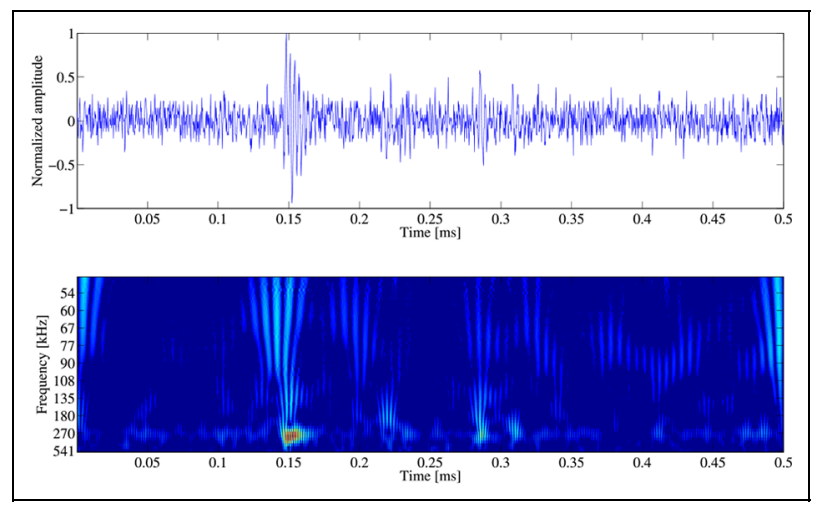

Figure 3. Time and time-frequency representation of fiber breakage events. frequency range of fiber breakage was reported by Bohse $^{32}$ at frequencies in the range of $[350 \mathrm{kHz}$, $700 \mathrm{kHz}$, whereas the lowest interval was reported as [300 kHz, $400 \mathrm{kHz}$ ] by Suzuki et al. ${ }^{33}$

\section{Time-frequency analysis}

The physical meaning, and hence interpretability of a measurement signal, is closely linked to the actual representation. Usually, damage information is not readily available from the time domain representation of a signal. ${ }^{34}$ Therefore, feature extraction is performed and damage-specific signatures are identified for diagnostic purposes. Furthermore, it can provide compression of the acquired data. Signal transforms, such as STFT, wavelet transform (WT), and HHT are mathematical methods which can be used to study AE signals in the frequency domain. ${ }^{10}$

In the context of this work, frequency domain features of transient AE bursts are determined by means of STFT, which provides acceptable data compression rates due to windowing. However, this method is limited by the trade-off between time and frequency resolution, which is related to the uncertainty principle. Here, the lower bound of time and frequency resolution is given as

$$
\Delta \tau \cdot \Delta w \geq \frac{1}{2}
$$

where $\Delta \tau$ and $\Delta w$ denote the time and frequency resolution, respectively. ${ }^{35}$ Increased window sizes lead to improvement in frequency resolution and decrease in time resolution. Furthermore, considering a particular window size, the time-frequency resolution is fixed.

\section{Pattern recognition}

The SVM is a supervised classification algorithm, which has emerged from the original research of Vapnik in the late 1970s. Due to its high accuracy and good generalization performance, ${ }^{36} \mathrm{SVM}$ is widely used in various pattern recognition tasks such as image classification, ${ }^{37}$ data mining, ${ }^{38}$ and classification of faults in rotating machinery. ${ }^{39}$ In this work, LIBSVM library is used for classification of $\mathrm{AE}$ waveforms. ${ }^{40}$

The goal of the training algorithm is to determine an optimal decision function in terms of a separating hyperplane, which geometrically separates different classes according to the training data. These data points, which are located closest to the separating hyperplane - referred to as support vectors - are of significant importance, because they "contain all the information to design the classifier." 41 To obtain a solution for training data, where different classes are 
not linearly separable, kernel functions are employed to perform a linear transformation of the feature space. ${ }^{36}$ From a practical point of view, Lin et al. advise using a radial basis function (RBF) kernel as the first choice. The stated reason is that there are fewer numerical difficulties to be faced compared to other kernel functions. ${ }^{42}$ In addition to class predictions, probability estimates are computed to assess the reliability of the results obtained from the deployed model using the implementation described by Chang and Lin. ${ }^{40}$ By default, the SVM provides only predictions of the class label based on the decision value $\hat{f}$ computed from the feature vector $x$. To obtain class conditional probabilities $p_{i}=P(y=i \mid x), \quad i=1, \ldots, k$, in a classification problem with $k$ classes, pairwise class probabilities $P(y=i \mid y=i o r j, x)$ are estimated as

$$
r_{i j} \approx \frac{1}{1+e^{A \hat{f}+B}}
$$

where $A$ and $B$ are determined according to training data. ${ }^{40}$ Finally, by solving the optimization problem

$$
\begin{aligned}
& \min _{p} \frac{1}{2} \sum_{i=1}^{k} \sum_{j: j \neq i}\left(r_{j i} p_{i}-r_{i j} p_{j}\right)^{2} \\
& \text { subject to } \quad p_{i} \geq 0, \forall i, \quad \sum_{i=1}^{k} p_{i}=1
\end{aligned}
$$

class conditional probabilities $p_{i}$ are obtained. ${ }^{40}$

\section{Results}

In this work, experiments were conducted to investigate the performance of the proposed classification scheme and related dependencies with respect to the load applied. To this end, AE measurements were conducted on multiple specimens, while they were subjected to cyclic loading patterns. Here, the loading of the structure promotes micro-mechanical fracture of the material as a result of damage propagation and, hence, activates characteristic AE source mechanisms. Statistical pattern recognition is employed to determine the underlying fracture mechanisms from the acquired $\mathrm{AE}$ waveforms. However, $\mathrm{AE}$ is an in situ inspection technique, that is, damage detection will only be possible on the initiation or propagation and is therefore non-deterministic. ${ }^{43}$ Moreover, considering structures under load, additional variability of the classification results may be related to the evolution of individual defects. Furthermore, loading conditions reportedly affect the activation of $\mathrm{AE}$ source mechanisms in aluminum specimens, which has already been found by Gagar et al. ${ }^{27}$ Therefore, measurements are performed at different points in time, while keeping the excitation motion constant to study the spread of classification performance over time due to statistical scattering and damage evolution. Moreover, measurements are performed using variable loading conditions as well as to investigate the impact of loading conditions on the classification performance.

A labeled dataset containing true class labels is constructed based on the results from several fracture tests. In particular, three-point bending as well as indentation flexure tests were employed. During each experiment, a large number of different AE signals can be detected, which are related to the fracture of the specimen. Reportedly, each of the damage modes can be identified in connection with three-point bending, ${ }^{10}$ whereas indentation flexure tests promote primarily delamination. ${ }^{29}$ From these experiments, several characteristic peak frequencies could be identified. The lowest characteristic frequency was assigned to delamination exhibiting peak frequencies in the spectrogram at approximately $45 \mathrm{kHz}$, which is in accordance with the findings of several authors. ${ }^{10,13}$ Furthermore, matrix crack is attributed to the peak frequencies of $95 \mathrm{kHz}$, which is in line with multiple reports from the literature. ${ }^{7,10,16,44}$ Moreover, debonding is presumably associated to the frequencies of approximately $245 \mathrm{kHz}$, which is in accordance with the literature. ${ }^{7,10,13,16}$ Finally, the maximum frequency of $300 \mathrm{kHz}$ is assigned to fiber breakage, which is located at the lower end of frequencies being reportedly related to fiber breakage. ${ }^{10,16,44}$ From each of the four classes, namely, (1) delamination, (2) matrix crack, (3) debonding, and (4) fiber breakage, 60 representative samples of AE were selected from a set of 13 fracture tests to construct a dataset for SVM training. Here, using a window size of 1024 data points, a set of 119 STFT coefficients between 40 and $500 \mathrm{kHz}$ are used as input to the classifier. The corresponding dataset is illustrated in Figure 4.

The classification performance of the SVM algorithm was evaluated using 10 -fold cross validation. Here, an RBF kernel was used as proposed by Lin et al., ${ }^{42}$ where the optimal classifier parameters $C$ and $\gamma$ were determined with respect to cross validation accuracy using grid search. Performance measures are summarized in Table 1. In general, good classification performance is achieved on this dataset.

During the cyclic loading experiments, couponshaped specimens with the dimensions $75 \mathrm{~mm} \times 175$ $\mathrm{mm} \times 1.8 \mathrm{~mm}$ were used. The specimens were manufactured from carbon fiber/epoxy composite material consisting of three layers of $\left[90^{\circ} / 0^{\circ} / 90^{\circ}\right]$ unidirectional layup patterns and two woven carbon/epoxy prepregs. Furthermore, similar initial damage was introduced to each specimen by means of three-point bending, because a strain threshold must be exerted to initiate AE activity in bending tests. ${ }^{17}$ Using carbon fiber/ 

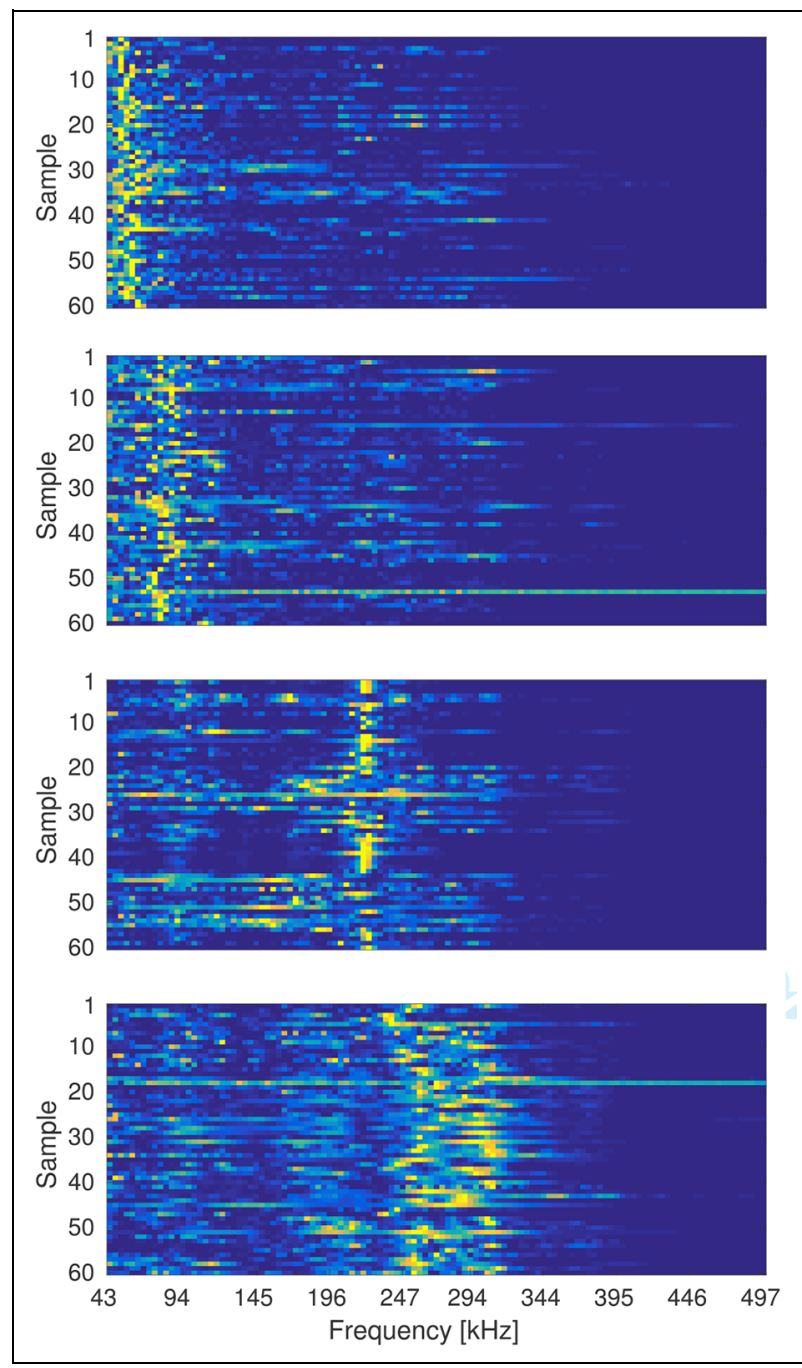

Figure 4. Visualization of the dataset used to build the classifier.

Table I. Cross validation performance.

\begin{tabular}{llll}
\hline Class & Accuracy & Specificity & Sensitivity \\
\hline Delamination & 0.94 & 0.85 & 0.96 \\
Matrix crack & 0.95 & 0.90 & 0.96 \\
Debonding & 0.94 & 0.87 & 0.96 \\
Fiber breakage & 0.97 & 0.93 & 0.98 \\
\hline
\end{tabular}

polymer composites, a significant fraction of the breaking load needs to be applied to give rise to micromechanical fracture due to the high bending elasticity of the material. According to Hamstad, ${ }^{45}$ only low AE activity is detected at $90 \%$ of breaking load if undamaged composite material is subjected to cyclic bending load. Therefore, split crack was introduced as initial damage prior to cyclic bending experiments.

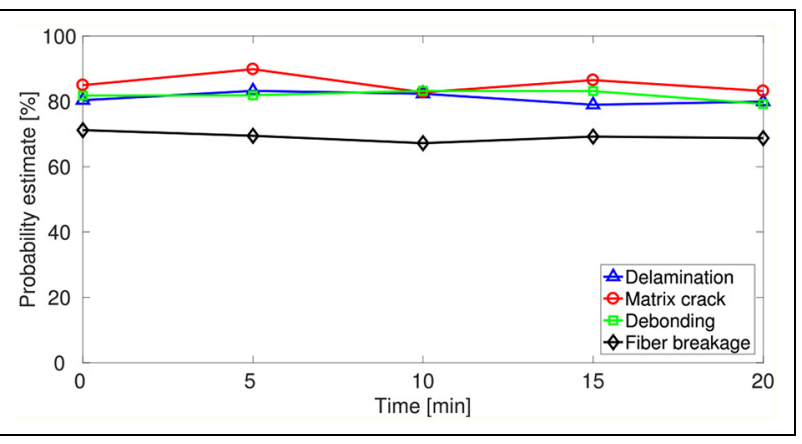

Figure 5. Mean values of probability estimation over time (I).

\section{Constant excitation}

To investigate the spread of the classification results over time, the excitation motion was kept constant and several measurements were performed at different points in time. Each series of measurements covers $20 \mathrm{~min}$ of time, while data acquisition was initiated every $5 \mathrm{~min}$ for a duration of $2 \mathrm{~s}$. Accordingly, five datasets were recorded per test series. The results of two measurement series subjecting a single specimen to two different excitation motions of (1) $[8 \mathrm{~mm}, 4 \mathrm{~Hz}]$ and (2) $[18 \mathrm{~mm}$, $5 \mathrm{~Hz}]$ are presented. Three classes (delamination, matrix crack, and debonding) are considered. To assess the reliability of the classification results, the mean values of the probability estimation are considered.

In Figure 5, results of the probability estimation which are related to excitation motion (1) are presented. This loading pattern is characterized by small amplitudes and intermediate frequency of cyclic load. In this example, probability estimates between $80 \%$ and $90 \%$ are achieved in most of the cases. The lowest probability estimates are obtained for matrix crack. The highest values of the probability estimation are frequently related to debonding. Furthermore, no significant changes in the probability estimates are apparent over the duration of the experiment.

Similar results were obtained using controlled excitation motion (2), which provides increased load intensity. The results are presented in Figure 6. Again, significant changes in the probability estimation could not be detected during the experiment. However, compared to the results of excitation (1), the overall classification performance could be improved using increased intensity of the loading pattern. This is especially noticeable in case of matrix crack. Furthermore, the highest probability estimation is now obtained for delamination in most of the cases, whereas the best results were usually obtained for debonding using lower load intensity (see Figure 5).

The main conclusion to be drawn from these experiments is that the results obtained remain constant over 


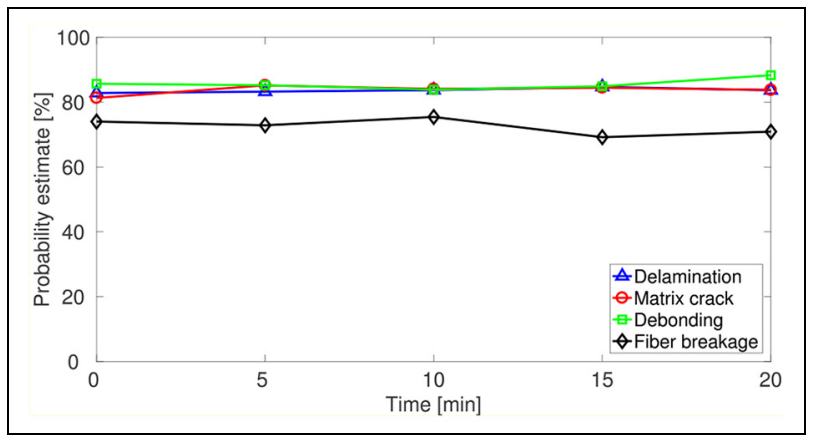

Figure 6. Mean values of probability estimation over time (2).

time. Only low scattering of the classification performance is observed among different points in time indicating that during operation and test time fundamental changes to the initial damage pattern due to, for example, crack development are not observed. This is important, because it excludes the related effects for the further experiment series to be reported in the sequel. However, an effect of the load intensity on the probability estimates is apparent. Generally, a slight improvement in the probability estimation of the classification results using higher load amplitude and frequency is evident. Also, the class with the highest probability estimation is different depending on the load intensity indicating the dependence of classification reliability on loading conditions. Therefore, a detailed investigation of the effect of loading conditions on the classification performance is presented.

\section{Variable excitations}

The evaluation of the classification performance presented in Table 1 is agnostic of environmental influences such as loading conditions, since they work on a finite set of example data. To investigate from a principle point of view the dependence of the classification reliability on the loading conditions, several specimens of composite material were subjected to different cyclic loading patterns. Each of the specimens is treated identically. Prior to the experiments, a split crack is introduced, so that mode I crack opening occurs as a result of bending load. To show that the reliability of the classification results typically varies depending on the loading conditions, three specimens S-I to S-III are selected.

During the experiments, AE measurements were performed while subjecting each of the specimens to any pair of the frequencies $[2 \mathrm{~Hz}, 3 \mathrm{~Hz}, 4 \mathrm{~Hz}, 5 \mathrm{~Hz}$, $6 \mathrm{~Hz}$ ] and amplitudes [6 mm, $9 \mathrm{~mm}, 12 \mathrm{~mm}, 15 \mathrm{~mm}$, $18 \mathrm{~mm}$. Hence, 25 datasets were acquired per specimen. During each measurement, data were acquired for $1.25 \mathrm{~s}$. Furthermore, each series of measurements follows the identical sequence. The first measurement was carried out using the lowest excitation amplitude and frequency of $[6 \mathrm{~mm}, 2 \mathrm{~Hz}]$. Hereafter, the frequency of the excitation motion was increased stepwise up to $6 \mathrm{~Hz}$ prior to increasing the excitation amplitude.

The classification results of each series of measurement performed on specimens S-I to S-III are summarized in Table 2. Similar to the previous experiments, the mean values of the probability estimation are computed from each dataset. From these results, contour plots are rendered to illustrate the dependence of probability estimation on the excitation motion. Here, the probability estimation is plotted on a color scale, while the $x$ - and $y$-axes denote the amplitude and frequency of the excitation motion, respectively. Damage detection with a high probability estimate is denoted by red shade. In case if no damage was detected, the probability estimation was set to 0 , which corresponds to dark blue shade.

Good results are generally achieved in connection with delamination. This damage mode is detected at any of the excitations. In many cases, high probability estimation is achieved. However, regions of excitation conditions (frequency, amplitude) leading to the best probability estimates differ among the specimens.

Regarding matrix crack, a strong dependency between loading patterns and classification results is apparent. Especially regarding specimen S-I, matrix crack could not be detected in several cases depending on the excitation. Considering specimens S-II and SIII, improvement of the probability estimates related to the classification results is observed on increasing load amplitudes. In contrast, using specimen S-I matrix crack is most frequently detected at small load amplitudes.

Considering debonding, the effect of excitation motion on the classification performance is more pronounced. Using each of the specimens, damage was not detected depending on the loading pattern. Especially in the case of S-I, debonding could only be detected during a single measurement. Similarly, improved damage detectability is observed on increasing load amplitudes. Considering specimens S-II and S-III, it is noticeable that the regions related to the best classification results are different among the specimens.

Fiber breakage could also be detected throughout each loading pattern. Analyzing the related probability estimations, improved results are frequently obtained for larger load amplitudes. However, using specimen S-III improved results at high frequency of the loading pattern. In contrast, improved probability estimation is obtained at low excitation frequencies in the case of S-I.

According to the experimental results presented in this section, strong dependences of (1) damage detectability and (2) the reliability of the classification result on excitation motion become evident. Whereas 


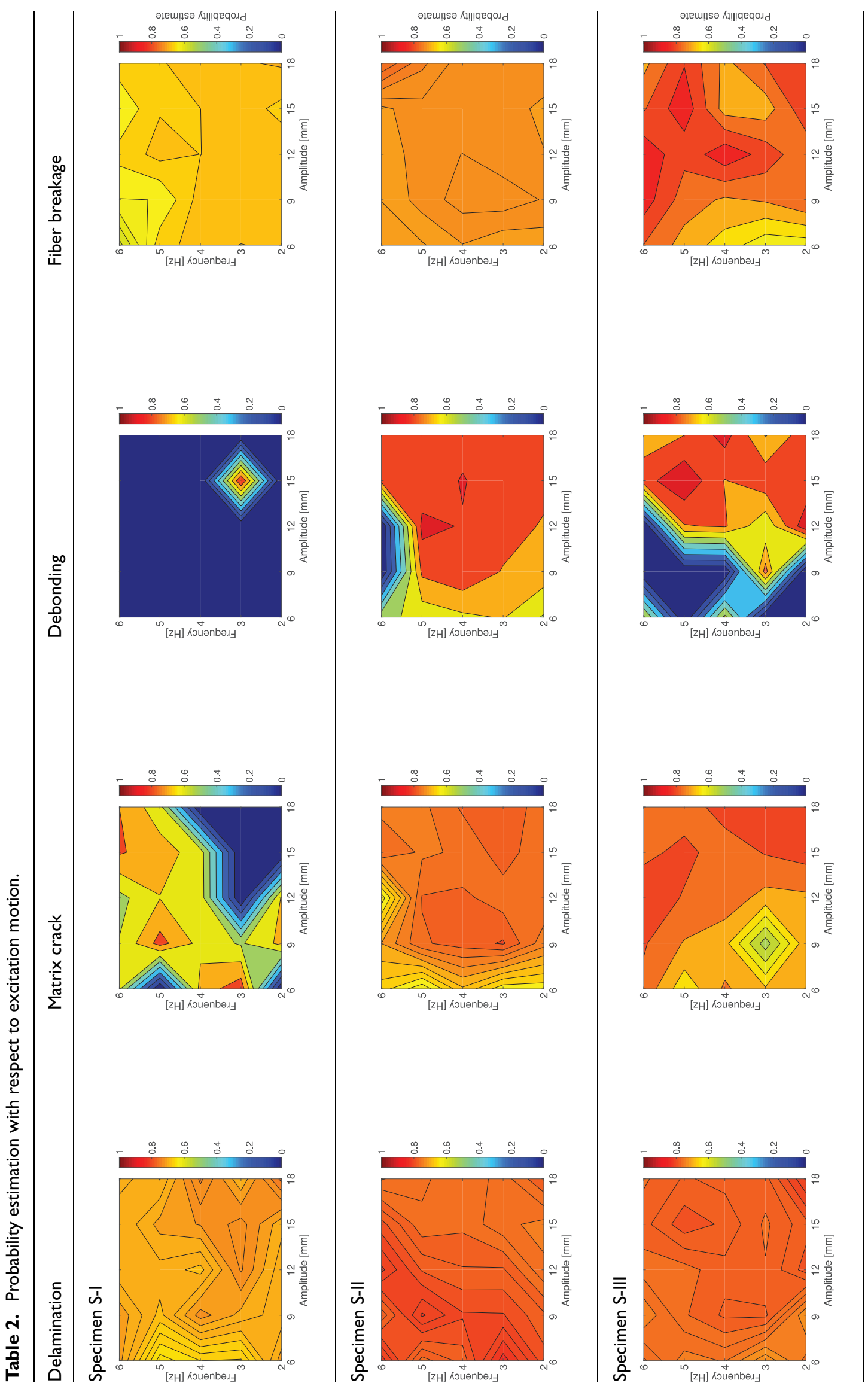


delamination and fiber breakage appear to be less sensitive to variable excitations, the classification results of matrix crack and debonding strongly vary with excitation motion. Due to a high degree of variability among the specimens and classes, a direct relationship between excitation motion and classification reliability could not be established. Nevertheless, cumulative trends are apparent. Frequently, increased load amplitudes lead to improvements in the detectability of damage and the corresponding probability estimate of the classification result. Also an increase in the frequency of the cyclic loading pattern leads to improved probability estimates in several cases. Similar findings are reported by the results of Gagar et al., ${ }^{27}$ in which influences on the activation of $\mathrm{AE}$ source mechanisms are investigated using different aluminum specimens under cyclic loading conditions. Here, large scattering of the AE waveform features under identical test conditions was observed. Furthermore, these results indicate cumulative trends in $\mathrm{AE}$ source activation with respect to loading conditions.

\section{Discussion}

Viewing the results considering the reliability of SHM systems, the question of which method can be used to evaluate the reliability of SHM systems rises. In the past, several ideas have been reported which address different aspects to adopt POD philosophy to SHM applications. For instance, in contrast to conventional NDT the results of SHM systems are statistically not independent due to high acquisition rates. ${ }^{21}$ In this context, Schubert Kabban et al. ${ }^{21}$ proposed a new methodology to adopt POD procedures to provide compatibility with dependent measurement data, which are obtained from SHM systems. Furthermore, multiple approaches developed to assess the reliability of SHM systems are summarized by Mandache et al. ${ }^{25}$ In particular, time-based POD is proposed to address the effect of damage evolution. ${ }^{25}$ It is suggested to find a formulation of the POD, which enables stating the probability of detecting specific defect growth within a given time interval. Multi-dimensional POD is proposed to take the effect of several in situ effects, that is, loading conditions, on SHM reliability into account. ${ }^{25}$ This includes the computation of POD with respect to each influencing factor to determine the actual reliability of the SHM system in particular situations. However, the approach requires availability of quantitative information on each influencing factor. Furthermore, quantitative knowledge regarding the impact of in situ effects on the reliability is necessary. In order to minimize the experimental effort required to determine POD, model-assisted approaches can be used. $^{20}$ Cobb et al. ${ }^{26}$ proposed a model-assisted approach for determining POD of crack detection in aluminum specimens using an in situ ultrasonic inspection technique. Moreover, Eckstein et al. ${ }^{46}$ proposed a methodology to quantify SHM performance using cumulative distribution functions to establish a probabilistic relationship between the detected and real damage size. From this representation, multiple metrics of SHM performance, such as minimum detectable damage size to define a lower bound of POD as accuracy of the inspection method, and the probability of false alarm are derived. However, identification of the underlying distribution functions is - particularly in the context of in situ inspection techniques, where a posteriori verification of real damage size is usually not possible - still an open issue.

From the aforementioned approaches to SHM reliability assessment, it is noticeable that the common weak point is characterized by missing detailed knowledge about the impact of different factors on SHM-related reliability properties. In this context, the experimentally shown results from the previous section state that the loading (which is unknown in practice) strongly affects the detectability of defects as well as the distinguishability of different damages. However, large scattering of the results prevents the establishment of a direct relationship, which strongly aggravates the online monitoring as well as the verification of healthy states.

\section{Summary and outlook}

Reliability assessment of the supervised SHM systems is an open issue which has to be solved before SHM comes into practice, especially regarding composite materials, which provide several advantages in many engineering applications. Currently, the more extensive use of composite material is restricted because safety and reliability requirements cannot be met due to complex damage modes. Due to its practical relevance, diagnosis regarding detection and discrimination of four different fracture mechanisms leading to failure composite material was chosen in this work as a showcase of SHM. The experimental results using a damage classification scheme were discussed with respect to their reliability.

To investigate influences on the classification reliability of deployed models, a mechanical test rig is used to subject specimens of composite material to various cyclic loading patterns. During loading of the specimens, AE measurements are performed. Furthermore, STFT and SVM are chosen as an example for the extraction of time-frequency domain features from time series data and classification of the measurement results. Two different types of experiments were 
performed. At first, constant excitations were used to assess the reproducibility of the classification results. Significant effects of damage evolution could not be detected, leading to the assumption that the test conditions are constant for the duration of the following experiments. Hereafter, a second series of experiments were performed using variable excitation motions. From the experimental results, it becomes evident that the performance of the classifier strongly depends on the excitation motion. However, a direct relationship could not be established due to large spreading of the classification results among multiple specimens of identical structure, partly leading to contradicting observations. Based on the chosen example related to fault detection and damage discrimination in CFRP material, the large scattering of the classification reliability under identical testing conditions is identified as a new scientific challenge in the context of reliability assessment of SHM systems.

\section{Declaration of conflicting interests}

The author(s) declared no potential conflicts of interest with respect to the research, authorship, and/or publication of this article.

\section{Funding}

The author(s) received no financial support for the research, authorship, and/or publication of this article.

\section{ORCID iD}

Sebastian Felix Wirtz (iD https://orcid.org/0000-0002-26380596

\section{References}

1. Pérez MA, Gil L and Oller S. Impact damage identification in composite laminates using vibration testing. Compos Struct 2014; 108: 267-276.

2. Agarwal BD, Broutman LJ and Chandrashekhara K. Analysis and performance of fiber composites. Hoboken, NJ: John Wiley \& Sons, 2006.

3. Crivelli D, Guagliano M, Eaton M, et al. Localisation and identification of fatigue matrix cracking and delamination in a carbon fibre panel by acoustic emission. Compos Part B: Eng 2015; 74: 1-12.

4. Farrar CR and Worden K. An introduction to structural health monitoring. Philos $T$ Roy Soc A 2007; 365: 303-315.

5. Gagar D, Martinez M and Foote P. Development of generic methodology for designing a structural health monitoring installation based on the acoustic emission technique. Proc CIRP 2014; 22: 103-108.

6. Miller RK and McIntire P. Nondestructive testing handbook: acoustic emission testing. Columbus, $\mathrm{OH}$ : American Society for Nondestructive Testing, 1987.
7. Marec A, Thomas JH and El Guerjouma R. Damage characterization of polymer-based composite materials: multivariable analysis and wavelet transform for clustering acoustic emission data. Mech Syst Signal Pr 2008; 22: 1441-1464.

8. Crivelli D, Guagliano M and Monici A. Development of an artificial neural network processing technique for the analysis of damage evolution in pultruded composites with acoustic emission. Compos Part B: Eng 2014; 56: 948-959.

9. De Oliveira R and Marques AT. Health monitoring of FRP using acoustic emission and artificial neural networks. Comput Struct 2008; 86: 367-373.

10. Hamdi SE, Le Duff A, Simon L, et al. Acoustic emission pattern recognition approach based on Hilbert-Huang transform for structural health monitoring in polymercomposite materials. Appl Acoust 2013; 74: 746-757.

11. McCrory JP, Al-Jumaili SK, Crivelli D, et al. Damage classification in carbon fibre composites using acoustic emission: a comparison of three techniques. Compos Part B: Eng 2015; 68: 424-430.

12. Prosser WH. Waveform analysis of AE in composites. In: Proceedings of the 6th international symposium on acoustic emission from composite materials, San Antonio, TX, 1-4 June 1998, pp. 61-70.

13. Gutkin R, Green CJ, Vangrattanachai S, et al. On acoustic emission for failure investigation in CFRP: pattern recognition and peak frequency analyses. Mech Syst Signal Pr 2011; 25: 1393-1407.

14. Liu PF, Chu JK, Liu YL, et al. A study on the failure mechanisms of carbon fiber/epoxy composite laminates using acoustic emission. Mater Design 2012; 37: 228-235.

15. Godin N, Huguet S and Gaertner R. Integration of the Kohonen's self-organising map and $k$-means algorithm for the segmentation of the AE data collected during tensile tests on cross-ply composites. NDT\&E Int 2005; 38: 299-309.

16. De Groot PJ, Wijnen PA and Janssen RB. Real-time frequency determination of acoustic emission for different fracture mechanisms in carbon/epoxy composites. Compos Sci Technol 1995; 55: 405-412.

17. Bussiba A, Kupiec M, Ifergane S, et al. Damage evolution and fracture events sequence in various composites by acoustic emission technique. Compos Sci Technol 2008; 68: 1144-1155.

18. Staszewski WJ. Intelligent signal processing for damage detection in composite materials. Compos Sci Technol 2002; 62: 941-950.

19. Das S, Srivastava AN and Chattopadhyay A. Classification of damage signatures in composite plates using oneclass SVMs. In: Proceedings of the IEEE aerospace conference, Big Sky, MT, 3-10 March 2007, pp. 1-19. New York: IEEE.

20. Kurz JH, Jüngert A, Dugan S, et al. Reliability considerations of NDT by probability of detection (POD) determination using ultrasound phased array. Eng Fail Anal 2013; 35: 609-617.

21. Schubert Kabban CM, Greenwell BM, DeSimio MP, et al. The probability of detection for structural health 
monitoring systems: repeated measures data. Struct Health Monit 2015; 14: 252-264.

22. Cai Y, Chow MY, Lu W, et al. Evaluation of distribution fault diagnosis algorithms using ROC curves. In: Proceedings of the IEEE power and energy society general meeting, Minneapolis, MN, 25-29 July 2010, pp. 1-6. New York: IEEE.

23. Majnik M and Bosnić Z. ROC analysis of classifiers in machine learning: a survey. Intell Data Anal 2013; 17 : 531-558.

24. Trafalis TB, Adrianto I and Richman MB. Active learning with support vector machines for tornado prediction. In: Proceedings of the international conference on computational science, Beijing, China, 27-30 May 2007, pp. 11301137. Berlin: Springer.

25. Mandache $\mathrm{C}$, Genest M, Khan M, et al. Considerations on structural health monitoring reliability. In: Proceedings of the international workshop smart materials, structures \& NDT in aerospace, Montreal, QC, Canada, 2-4 November 2011.

26. Cobb AC, Fisher J and Michaels J. Model-assisted probability of detection for ultrasonic structural health monitoring. In: Proceedings of the 4th European-American workshop on reliability of NDE, Berlin, 24-29 June 2009.

27. Gagar D, Foote P and Irving P. Effects of loading and sample geometry on acoustic emission generation during fatigue crack growth: implications for structural health monitoring. Int J Fatigue 2015; 81: 117-127.

28. Scruby CB. An introduction to acoustic emission. $J$ Phys E Sci Instrum 2000; 20: 946-953.

29. Al-Jumaili SK, Holford KM, Eaton MJ, et al. Parameter Correction Technique (PCT): a novel method for acoustic emission characterisation in large-scale composites. Compos Part B: Eng 2015; 75: 336-344.

30. Fasana A and Garibaldi L. Measurement of acoustic emission signals: influence of the couplant. Key Eng Mater 2007; 347: 375-380.

31. Theobald PD, Zeqiri B and Avison J. Couplants and their influence on AE sensor sensitivity. J Acoust Emiss 2008; 26: 91-97.

32. Bohse J. Acoustic emission characteristics of microfailure processes in polymer blends and composites. Compos Sci Technol 2000; 60: 1213-1226.

33. Suzuki M, Nakanishi H, Iwamoto M, et al. Application of static fracture mechanisms to fatigue fracture behavior of class A-SMC composite. In: 4th Japan-US conference on composite materials, Washington, DC, 1989, pp. 297-306.

34. Worden K, Farrar CR, Manson G, et al. The fundamental axioms of structural health monitoring. $P$ Roy Soc A: Math Phy 2007; 463: 1639-1664.

35. Mertins A. Signaltheorie. Wiesbaden: Springer Verlag, 2013.

36. Burges CJC. A tutorial on support vector machines for pattern recognition. Data Min Knowl Disc 1998; 2: 121-167.

37. Chaplot S, Patnaik LM and Jagannathan NR. Classification of magnetic resonance brain images using wavelets as input to support vector machine and neural network. Biomed Signal Proces 2006; 1: 86-92.

38. Tan KC, Teoh EJ, Yu Q, et al. A hybrid evolutionary algorithm for attribute selection in data mining. Expert Syst Appl 2009; 36: 8616-8630.

39. Samanta B. Gear fault detection using artificial neural networks and support vector machines with genetic algorithms. Mech Syst Signal Pr 2004; 18: 625-644.

40. Chang CC and Lin CJ. LIBSVM: a library for support vector machines. ACM T Intel Syst Tec 2011; 2(3): 27:1-27: 27.

41. Yin Z and Hou J. Recent advances on SVM based fault diagnosis and process monitoring in complicated industrial processes. Neurocomputing 2016; 174: 643-650.

42. Lin CJ, Hsu CW and Chang CC. A practical guide to support vector classification. Technical report, Department of Computer Science, National Taiwan University, Taipei, Taiwan, 2003.

43. Pollock A. Probability of detection for acoustic emission. $J$ Acoust Emiss 2007; 25: 231-237.

44. Bak KM, KalaiChelvan K, Vijayaraghavan GK, et al. Acoustic emission wavelet transform on adhesively bonded single-lap joints of composite laminate during tensile test. J Reinf Plast Comp 2012; 32: 87-95.

45. Hamstad MA. A review: acoustic emission, a tool for composite-materials studies. Exp Mech 1986; 26: 7-13.

46. Eckstein B, Fritzen CP and Bach M. Considerations on the reliability of guided ultrasonic wave-based SHM systems for CFRP aerospace structures. In: Proceedings of the 6th European workshop on structural health monitoring, Dresden, 3-6 July 2012. 


\section{DuEPublico}

UNIVERSITAT
DUUISBU R G

Duisburg-Essen Publications online

offen im Denken

$\mathbf{U b} \mid \begin{aligned} & \text { universitäts } \\ & \text { bibliothek }\end{aligned}$

This text is made available via DuEPublico, the institutional repository of the University of Duisburg-Essen. This version may eventually differ from another version distributed by a commercial publisher.

DOI: $\quad 10.1177 / 1475921718791894$

URN: urn:nbn:de:hbz:464-20210506-104048-7

This publication is with permission of the rights owner freely accessible due to an Alliance licence and a national licence (funded by the DFG, German Research Foundation) respectively.

(C) The Author(s) 2018. All rights reserved. 